Vol. 6, No. 2, 2020

Ihor Vrublevskyi

Department of Engineering Mechanics (Weapons and Equipment of Military Engineering Forces), Hetman Petro Sahaidachnyi National Army Academy, Ukraine, Lviv, 32 Heroes of Maidan Street,

E-mail: vrublew@gmail.com, ORCID 0000-0001-5954-7754

\title{
OPTIMIZATION OF VIBRATORY CONVEYING UPWARD BY INCLINED TRACK WITH POLYHARMONIC NORMAL VIBRATIONS
}

\author{
Received: May 12, 2020 / Revised: June 29, 2020 / Accepted: September 30, 2020
}

(C) Vrublevskyi I., 2020

\begin{abstract}
The paper is devoted to the research of vibratory conveying of piece goods along an inclined track, performing harmonic longitudinal and polyharmonic normal vibrations. It is considered the conditions of reaching maximum conveying velocity at specified values of frequency and amplitude of longitudinal vibrations - the conditions of maximum dimensionless conveying velocity, depending on several dimensionless parameters in the moving modes without hopping. These dimensionless parameters are the inclination angle parameter - a ratio of an inclination angle tangent to a frictional coefficient, the intensive vibration coefficient - a ratio of the longitudinal amplitude of vibration to the amplitude of the first harmonic of normal vibration and frictional coefficient. Maximal conveying velocity is achieved at the certain values of normal vibration amplitudes and values of phase difference angles between longitudinal and normal vibrations, which are called optimal, and their values are dependent on these two dimensionless parameters, while maximum normal vibration acceleration should be equal to the gravitational acceleration. The research was made by approximate harmonic balance method and by numerical step-by-step integration method, which allows performing calculations with any given accuracy. The results obtained by the two methods are compared.

To determine the maximal and optimal values of elevation angles, there are calculated the maximal value of the inclination angle parameter at which the value of dimensionless velocity is equal to zero, and the value of the inclination angle parameter at which a particle moves to a specified height in the minimum time. The optimal values of amplitudes of harmonics of polyharmonic normal vibration are determined in dependence on the inclination angle parameter with the number of harmonics from four to seven. The graphs of these dependencies are presented and the most important values of dimensionless parameters are presented in the table.
\end{abstract}

Keywords: vibratory conveying, polyharmonic normal vibration, harmonic longitudinal vibration, electromagnetic drive.

\section{Introduction}

Vibratory conveying devices are widely used in different industrial enterprises, especially for parts feeding, orienting, or internal transportation in automated assembly systems, robotized industrial production lines [1], [2]. For these purposes, the most popular devices are vibratory conveyors, bowl feeders, vibratory manipulators with electromagnetic drive due to their high reliability and productivity, design's simplicity. They must transport parts not only horizontally, but also along an inclined plane or a spiral trough upwards. The most common and simple vibratory conveying devices provide the linear vibration of conveying track, that is suitable for conveying of relatively small and not very massive piece goods. For conveying the massive heavy goods or fragile and explosive products, it is necessary to ensure the moving of products without hopping. However, the vibratory conveying devices with linear vibration do not allow achieving high conveying velocity and large elevation angles of conveying track in the 
moving modes without hopping. The increasing of conveying velocity and elevation angles in non-hopping modes, and as a result the increase of productivity, can be reached in vibratory devices with asymmetric longitudinal vibration or with independent longitudinal and normal vibrations of conveying track.

\section{Problem statement}

For reaching the high conveying velocity, the conveying devices with electromagnetic drives should provide large amplitude of longitudinal vibrations. That is why the electromagnetic drive of longitudinal vibration should operate in near-resonant mode, in which vibration is sinusoidal. At the same time, the amplitude of normal vibration should be stable to ensure the moving without hopping, and the electromagnetic drive of normal vibration should be operated far from resonance, where vibration can be non-sinusoidal, in particular polyharmonic. In author's papers [3], [4] there was considered vibratory conveying by harmonic longitudinal and polyharmonic normal vibrations, represented by equations:

$$
\begin{gathered}
x=A \sin (\omega t+\varepsilon), \\
y=\sum_{i=1}^{n} A_{i} \sin \left[\left(i \omega t+\frac{\pi}{2}(i-1)\right],\right.
\end{gathered}
$$

where $A$ - amplitude of longitudinal vibration, $A_{i}$ - amplitude of $i$-th harmonic of normal vibration, $\omega-$ frequency of vibration, $n$ - number of harmonics, $\varepsilon$ - phase difference angle, $t$ - time.

These studies were carried out by approximate harmonic balance method, and their results are reliable when longitudinal vibration amplitude is essentially larger than normal vibration amplitudes. The numerical methods of investigation, for example step-by-step integration method, using the methods solving of nonlinear equations, provide the possibility to determine the optimal parameters of vibrations to reach the maximal velocity for any cases. It was found that the maximal conveying velocity is achieved at a certain ratio of amplitudes of normal vibration's harmonics. Moreover, this ratio, which was called optimal, depends on the inclination angle of conveying track. However, the dependence of the optimal ratio of normal vibration's harmonics on inclination angles was researched for vibration with two and three harmonics only.

\section{Review of Modern Information Sources on the Subject of the Paper}

The investigation of vibratory conveying of a material point by two-component independent longitudinal and normal vibrations has been made in many papers. In particular, the simplest case -elliptical vibration harmonic longitudinal and normal vibrations with a phase difference between them, when conveying track vibrates by elliptical trajectory, is considered in [1], [5]-[7]. It was proved that elliptical vibration can achieve higher conveying velocity and elevation angles comparing to linear vibration, especially in nonjumping modes of moving. An even higher value of velocity and elevation angles can be reached by using polyharmonic vibration, in particular biharmonic vibration. Vibratory conveying by biharmonic longitudinal vibration is considered in [2], [8], but conveying velocity, in this case, is much less than in the case of two-component independent vibrations. Vibratory conveying by two-component biharmonic vibrations was considered in [9], conveying by biharmonic normal vibration with three harmonics was considered in [10], but optimal parameters of vibration were not determined here. The optimal relative to velocity law of two-component vibrations with a limited value of acceleration has obtained by Lavendel [11]. According to this law, longitudinal and normal vibrations should be functions with piecewise constant acceleration. Nevertheless, this law is difficult to implement by an electromagnetic drive.

The author's research [3,4] shows that using harmonic longitudinal and polyharmonic normal vibrations can sufficiently increase conveying velocity and elevation angles at certain ratios of amplitudes and phase differences angles.

\section{Objectives and Problems of Research}

The mean conveying velocity $v$ can be calculated by formula [2]:

$$
v=A \omega V \text {, }
$$

where $V$ - dimensionless conveying velocity - parameter, depending on several dimensionless parameters. The effectiveness of the process of vibratory conveying can be estimated by the value of $V$, which determines a velocity per unit frequency and amplitude of longitudinal vibration. 


\section{Ihor Vrublevskyi}

In this paper, the dependence of dimensionless conveying velocity on dimensionless vibration parameters with harmonic longitudinal and polyharmonic normal vibrations with the number of harmonics from 2 to 7 is investigated. It is shown that maximal conveying velocity can be reached with the definite values of normal harmonics ratio, which depend on the inclination angle of conveying track. The dependencies of dimensionless conveying velocity on dimensionless vibration parameters are investigated by numerical methods that ensure the specified accuracy. The optimal values of the ratio of harmonic amplitudes of normal vibrations and the values of maximal and optimal elevation angles are researched.

\section{Optimization of conveying velocity by harmonic balance method}

According to the investigations of two-component vibratory conveying $[2,3,5]$, the value of dimensionless $V$ velocity depends on the next dimensionless parameters: $K_{\alpha}$ - the inclination angle parameter, $K_{\beta}$ - the intensive vibration parameter, $G$ - the gravitational overload parameter, which are calculated as follows:

$$
K_{\alpha}=\frac{\tan \alpha}{\mu}, \quad \quad K_{\beta}=\frac{A}{A_{1} \mu}, \quad G=\frac{A_{m} \omega^{2}}{g \cos \alpha},
$$

where $g$ - the gravitational acceleration, $A_{m}$ - amplitude of normal vibration in a moment when its acceleration is maximum.

The harmonic balance method allows determining the optimal values of amplitudes of harmonics acceleration $w_{i}$ with sufficient accuracy only with a fairly sufficiently intense longitudinal vibration, when $A_{x} \gg A_{m}$ or $K_{\beta} \rightarrow \infty$. In this case, according to the investigations [2], the particles move in the mode with one sliding motion upward and one sliding motion downward during the vibration period without sticks relative to the track. The optimal phase difference angle $\varepsilon=90^{\circ}$ and amplitudes of harmonic acceleration $w_{i}$ should satisfy the equation [3]:

$$
1+\Theta-\frac{2}{\pi}\left(\psi+\sum_{i=1}^{n} \frac{w_{i}}{i} \sin i \psi\right)=0
$$

where $\psi=\left(2 \pi+\tau_{+}-\tau_{-}\right) / 2 ; \quad w_{i}=\frac{i^{2} A_{i} \omega^{2}}{g \cos \alpha}-$ dimensionless normal vibration acceleration amplitude of $i$-th harmonic; $\tau=\tau_{+}$and $\tau=\tau_{-}-$the initial moments of the sliding motion upward and sliding motion downward respectively, $\tau=\omega t$.

The conditions of a particles moving without hopping with maximum velocity are described by the system of equations:

$$
\begin{aligned}
& \sum_{i=1}^{n} w_{i} \sin \left(i \tau_{m}+\frac{\pi(i-1)}{2}\right)=1 ; \\
& \sum_{i=1}^{n} i w_{i} \cos \left(i \tau_{m}+\frac{\pi(i-1)}{2}\right)=0,
\end{aligned}
$$

where $\tau_{m}$ - the moment of maximum normal acceleration.

The dimensionless velocity in this case is calculated by formula [3]:

$$
V=-\cos (\psi) \text {. }
$$

The optimal values of amplitudes $w_{i}$ can be determined by the method of Lagrange multipliers with the additional $n$ equations:

$$
\frac{1}{i} \sin i \psi+\lambda\left[i \cos \left(i \tau_{m}+\frac{\pi(i-1)}{2}\right)-i^{2} \sin \left(i \tau_{m}+\frac{\pi(i-1)}{2}\right)\right]=0,
$$

where $\lambda$ - unknown Lagrange multiplier.

The Eqs. (3), (4), (6), (7) form the system of $n+3$ equations with $n+3$ unknown variables $\sum_{i=1}^{n} w_{i}, \psi$, $\tau_{m}, \lambda$. However, this system of equations can have many solutions, to choose a desirable solution, it is necessary to introduce the additional inequalities, for example, $w_{1}<2 ; w_{2}>w_{3} ; 0<\tau_{m}<\pi / 2$, etc. 


\section{Investigation of dimensionless velocity $V$ dependence on dimensionless parameters by the step-by-step integration method}

The equation of a particle's motion without hopping on a track, vibrating according to Eqs. (1), using the values dimensionless distance $\chi=x / A$, can be represented by analogy with the equation describing the non-hopping motion with elliptical vibration [12], as follows:

$$
\chi^{\prime \prime}=l_{ \pm} \sin \left(\tau-\kappa_{ \pm}\right)-\delta_{ \pm}+\sum_{i=2}^{n} \frac{w_{i}}{w_{1} q_{ \pm}} \sin \left\{i \tau-\left[i \varepsilon-\frac{\pi}{2}(i-1)\right]\right\},
$$

where $l_{ \pm}=\frac{\sqrt{1+K_{\beta}^{2} \pm 2 K_{\beta} \cos \varepsilon}}{K_{\beta}} ; \quad \kappa_{ \pm}=\arctan \frac{\sin \mathcal{E}}{\cos \varepsilon \pm K_{\beta}} ; \quad \delta_{ \pm}=\frac{\Theta \pm 1}{K_{\beta} w_{1} l_{ \pm}} ; \quad q_{ \pm}=K_{\beta} l_{ \pm}$. The signs "+" and "-" correspond to the particle sliding upward and downward respectively.

The initial moments of the sliding motion upward $\tau=\tau_{+}$and sliding motion downward $\tau=\tau_{-}$are determined as the roots of the system of two nonlinear equations:

$$
\begin{gathered}
\cos \left(\tau_{+}-\kappa_{+}\right)-\cos \left(\tau_{-}-\kappa_{+}\right)+\delta_{+}\left(\tau_{+}-\tau_{-}\right)+ \\
+\sum_{i=2}^{n} \frac{w_{i}}{i w_{1} q_{+}}\left\{\cos \left[i \tau_{+}-\left(i \varepsilon-\frac{\pi(i-1)}{2}\right)\right]-\cos \left[i \tau_{-}-\left(i \varepsilon-\frac{\pi(i-1)}{2}\right)\right]\right\}=0 \\
\cos \left(\tau_{-}-\kappa_{-}\right)-\cos \left(\tau_{+}-\kappa_{-}\right)+\delta_{-}\left(2 \pi+\tau_{-}-\tau_{+}\right)+ \\
+\sum_{i=2}^{n} \frac{w_{i}}{i w_{1} q_{-}}\left\{\cos \left[i \tau_{-}+\left(i \varepsilon-\frac{\pi(i-1)}{2}\right)\right]-\cos \left[i \tau_{+}+\left(i \varepsilon-\frac{\pi(i-1)}{2}\right)\right]\right\}=0 .
\end{gathered}
$$

Dimensionless velocity can be found by formula:

$$
V=\frac{S_{+}+S_{-}}{2 \pi},
$$

where $S_{+}$and $S_{-}-$dimensionless distances of a particle moving upward and backward respectively during the vibration period, that are calculated by equations:

$$
\begin{gathered}
S_{+}=l_{+}\left\{\cos \left(\tau_{+}-\kappa_{+}\right)\left(\tau_{-}-\tau_{+}\right)+\sin \left(\tau_{+}-\kappa_{+}\right)-\sin \left(\tau_{-}-\kappa_{+}\right)-\delta_{+}\left(\tau_{-}-\tau_{+}\right)^{2} / 2+\right. \\
+\sum_{i=2}^{n} \frac{w_{i}}{i^{2} w_{1} q_{+}}\left\{i \cos \left[i \tau_{+}-\left(i \varepsilon-\frac{\pi(i-1)}{2}\right)\right]\left(\tau_{-}-\tau_{+}\right)-\right. \\
\left.-\sin \left[i \tau_{-}-\left(i \varepsilon-\frac{\pi(i-1)}{2}\right)-\sin \left[i \tau_{+}-\left(i \varepsilon-\frac{\pi(i-1)}{2}\right)\right]\right\}\right\} ; \\
S_{-}=l_{-}\left\{\cos \left(\tau_{-}-\kappa_{-}\right)\left(\tau_{+}-\tau_{-}\right)+\sin \left(\tau_{-}-\kappa_{-}\right)-\sin \left(\tau_{+}-\kappa_{-}\right)-\delta_{-}\left(2 \pi+\tau_{+}-\tau_{-}\right)^{2} / 2+\right. \\
+\sum_{i=2}^{n} \frac{w_{i}}{i^{2} w_{1} q_{-}}\left\{i \cos \left[i \tau_{-}+\left(i \varepsilon-\frac{\pi(i-1)}{2}\right)\right]\left(2 \pi+\tau_{+}-\tau_{-}\right)-\right. \\
\left.-\sin \left[i \tau_{+}+\left(i \varepsilon-\frac{\pi(i-1)}{2}\right)-\sin \left[i \tau_{-}+\left(i \varepsilon-\frac{\pi(i-1)}{2}\right)\right]\right\}\right\} .
\end{gathered}
$$

Having given the values parameters $K_{\alpha}$ and $K_{\beta}$ and a set of dimensionless amplitudes of harmonics acceleration $w_{i}$, satisfying the condition $G=1$, substituting them into Eq. (8), we calculate the values of $l_{ \pm}$, $\kappa_{ \pm}, \delta_{ \pm}, q_{ \pm}$. Then we determine the roots $\tau_{+}$and $\tau_{-}$of system Eqs. (9) and (10) in the range from 0 to $2 \pi$, changing the value of the angle $\varepsilon$ from $60^{\circ}$ to $90^{\circ}$. Then we substitute the values of $\tau_{+}$and $\tau_{-}$into Eqs. (12)

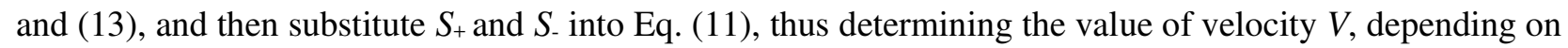
parameters $K_{\alpha}$ and $K_{\beta}$ and the optimal values of $w_{i}$ and $\varepsilon$, providing a maximum of $V$.

\section{Dependence of dimensionless velocity $V$ and optimal dimensionless amplitudes of harmonics $w_{i}$ on inclination angle parameter $K_{\alpha}$}

Dependence of dimensionless velocity $V$ on the inclination angle parameter $K_{\alpha}$ was studied by harmonic balance method in [3], the graphs of dependence $V\left(K_{\alpha}\right)$ were presented for the number of 


\section{Ihor Vrublevskyi}

harmonics $n=1-7$. However, the dependence of $w_{i}$ on parameter $K_{\alpha}$ has been not studied for $n>3$. That is why the values of $V$ at $K_{\alpha}>0.7$ and $n \geq 4$ are incorrect and somewhat underestimated.

The dependence of velocity $V$ on parameters $K_{\alpha}$ and $K_{\beta}$ was studied by the harmonic balance method and the step-by-step integration method. The approximated harmonic balance method gives accepted results, comparable to the results of step-by-step integration method, for the values $K_{\beta}>40 \ldots 50$.

Calculations were performed using MathCAD. The dependencies of dimensionless velocity $V$ on inclination angle parameter $K_{\alpha}$ for number of harmonics $n=1-7$ are shown in Fig. 1. Dimensionless velocity $V$ increases with the increasing number of harmonics $n$ and decreases with increasing of inclination angle parameter.

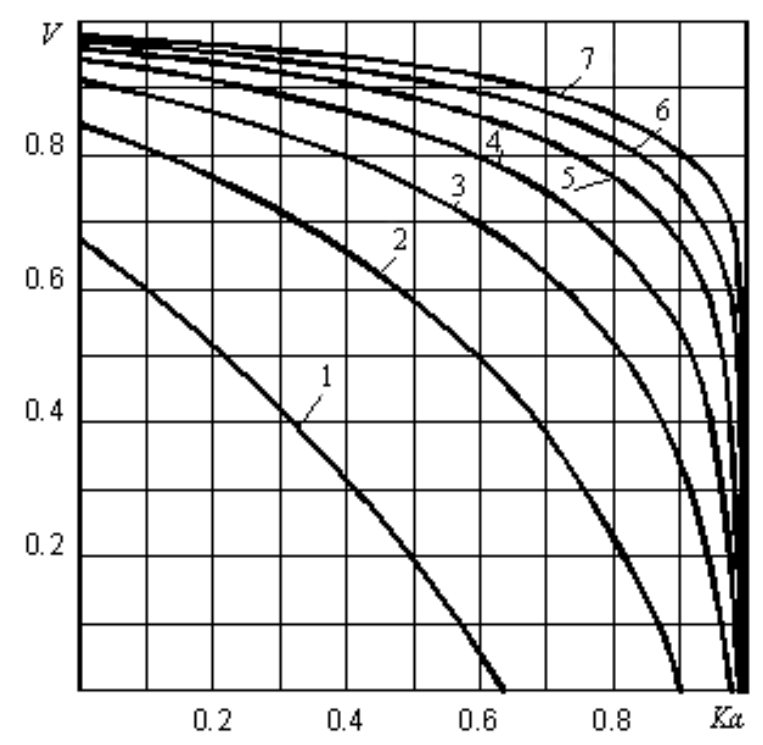

Fig. 1. Dependence of dimensionless velocity $V$ on inclination angle parameter $K_{\alpha}$ with values of parameters $K_{\beta} \rightarrow \infty, G=1$ and different number of harmonics $n$ from 1 to 7

Graphs of optimal amplitudes $w_{i}$ dependencies on parameters $K_{\alpha}$ for numbers of harmonics $n=2$ and 3 were determined in the author's works [13]. Graphs of such dependencies for $n>3$ are shown in Fig. 2. Continuous lines in Fig. $2 a$ show graphs for $n=4$ and dashed lines show graphs for $n=5$; continuous lines on Fig. $2 b$ show graphs for $n=6$ and dashed lines show graphs for $n=7$. These results were obtained by an approximate harmonic balance method, they are valid for $G=1 ; K_{\beta} \rightarrow \infty$. As seen in Fig. 2, with increasing of $K_{\alpha}$ the optimal values of the lower harmonics $(i \leq n / 2)$ amplitudes increase but the values of higher harmonics $(i>n / 2)$ amplitudes decrease. However, at the range $K_{\alpha}>0.9$ optimal amplitudes of all harmonics decrease sharply with increasing of $K_{\alpha}$.

The values of dimensionless velocity $V$ for horizontal conveying $\left(K_{\alpha}=0\right)$ with intensive vibration $\left(G=1, K_{\beta}>40 \ldots 50\right)$ with optimal ratio of the amplitudes of harmonics with their number from 1 to 7 and with optimal difference phase angles are shown in Table 1.

To determine the value of dimensionless velocity $V$ at various values of $K_{\beta}$, the step-by-step integration method should be applied, which allows obtaining the result with a given accuracy.

Table 1

Maximal dimensionless velocity and optimal normal acceleration amplitudes

\begin{tabular}{|c|c|c|c|c|c|c|c|c|}
\hline Number of harmonics $n$ & $V\left(\right.$ at $\left.K_{\alpha}=0\right)$ & $w_{1}$ & $w_{2}$ & $w_{3}$ & $w_{4}$ & $w_{5}$ & $w_{6}$ & $w_{7}$ \\
\hline 1 & 0.6736 & 1 & - & - & - & - & - & - \\
\hline 2 & 0.8469 & 1.355 & 0.643 & - & - & - & - & - \\
\hline 3 & 0.9122 & 1.527 & 0.998 & 0.471 & - & - & - & - \\
\hline 4 & 0.9433 & 1.628 & 1.213 & 0.783 & 0.370 & - & - & - \\
\hline 5 & 0.9604 & 1.694 & 1.354 & 0.998 & 0.643 & 0.329 & - & - \\
\hline 6 & 0.9709 & 1.740 & 1.454 & 1.152 & 0.844 & 0.543 & 0.258 & - \\
\hline 7 & 0.9776 & 1.774 & 1.527 & 1.266 & 0.998 & 0.730 & 0.470 & 0.225 \\
\hline
\end{tabular}



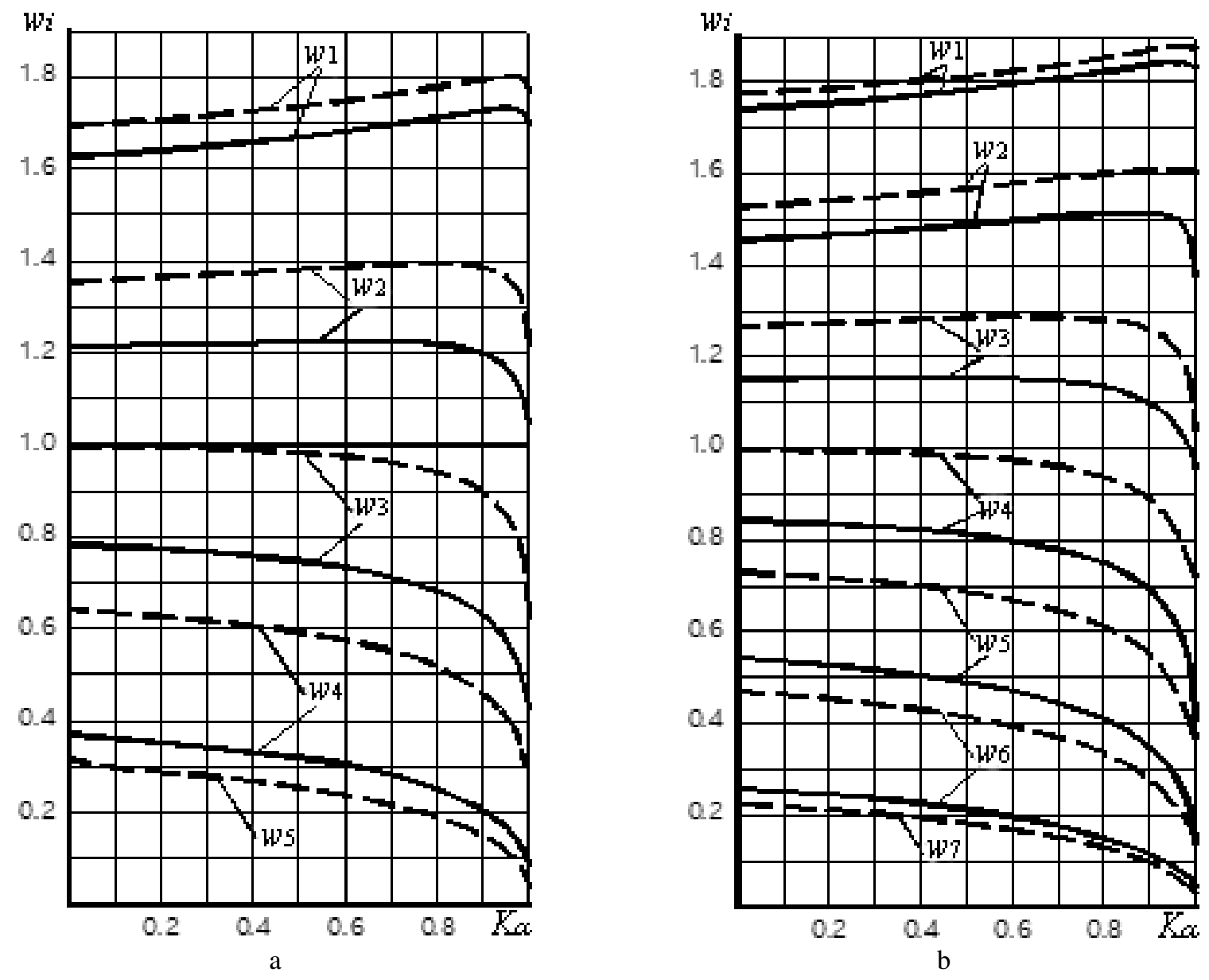

Fig. 2. Dependence of optimal amplitudes of dimensionless normal acceleration $w_{i}$ on inclination angle parameter $K_{\alpha}$ (when parameters $K_{\beta} \rightarrow \infty, G=1$ ) with different number of harmonics: $\mathrm{a}-n=4$ - continuous lines, $n=5$ - dashed lines; $\mathrm{b}-n=6$ - continuous lines, $n=7-$ dashed lines

\section{Dependence of dimensionless velocity $V$ on parameter $K_{\beta}$}

The step-by-step integration method allows calculating the dependence of dimensionless velocity $V$ on parameters $K_{\alpha}$ and $K_{\beta}$ with different values of amplitudes $w_{i}$ and angle $\varepsilon$. The initial values of $w_{i}$ were taken from approximate results by harmonic balance method (Fig. 2) for specific values of $K_{\alpha}$ and $n$, and varied in small intervals, observing the condition $G=1$. The value of angle $\varepsilon$ varied from $60^{\circ}$ to $90^{\circ}$. For the calculations, a MathCAD-program was developed, at the input of which the values of parameters $K_{\beta}$, $K_{\alpha}, n$, initial values of $w_{i}$ and $\varepsilon$ were set, and at the output, the value of $V$ have obtained, as well as the optimal values of $w_{i}$ and $\varepsilon$.

The dependence of velocity $V$ on parameter $K_{\beta}$ at $K_{\alpha}=0$ (the optimal horizontal conveying without hopping) with different numbers of harmonics of normal vibrations $n$ from 2 to 7 and optimal values of amplitudes $w_{i}$ and angle $\varepsilon$, is shown in Fig. 3 by continuous lines. Similar dependence at $K_{\alpha}=0.9$ (with inclinations angles close to the limiting values) is shown by dashed lines.

An important property of vibratory conveying of a material point with two-component vibration of conveying track is that the dimensionless velocity $V$ is practically independent on $K_{\beta}$ with an increase of $K_{\beta}$, starting from $K_{\beta}>40 \ldots 50$. The optimal value of angle $\varepsilon$ depends on the values of parameters $K_{\alpha}$ and $K_{\beta}$.

\section{Determining of limiting and optimal values of inclination angle parameter $\boldsymbol{K}_{\alpha}$}

The limiting value of inclination angle parameter $K_{\alpha}=K_{\alpha}$ lim is the maximal value of $K_{\alpha}$ at which dimensionless velocity $V$ equals zero. The value of $K_{\alpha} \lim$ at the number of harmonics $n=2$ and $n=3$ are determined in [4]. When $n>4$ the value of $K_{\alpha} \lim \rightarrow 1$, i.e. the inclination angle becomes equal to the angle 


\section{Ihor Vrublevskyi}

of friction. However, the use of angles corresponding to $K_{\alpha}>0.9$ is undesirable, since the value of the friction coefficient is not always accurately known, besides, it may change during conveying and particles cannot slide upward. The values of parameter $K_{\alpha}$ lim for different numbers of harmonics $n$ are given in Table 2 .

Table 2

The limited and optimal values of the inclination angle parameter $K_{\alpha}$

\begin{tabular}{|c|c|c|c|c|}
\hline Number of harmonics $n$ & $K_{\alpha \text { lim }}$ & $K_{\alpha \text { opt }}$ & $V\left(\right.$ at $\left.K_{\alpha}=K_{\alpha \text { opt }}\right)$ & $V K_{\alpha o p t}$ \\
\hline 1 & 0.636 & 0.35 & 0.37 & 0.129 \\
\hline 2 & 0.900 & 0.57 & 0.524 & 0.299 \\
\hline 3 & 0.977 & 0.71 & 0.614 & 0.436 \\
\hline 4 & 0.996 & 0.79 & 0.634 & 0.540 \\
\hline 5 & 0.999 & 0.84 & 0.736 & 0.618 \\
\hline 6 & 0.999 & 0.87 & 0.779 & 0.678 \\
\hline 7 & 0.999 & 0.90 & 0.807 & 0.726 \\
\hline
\end{tabular}

The optimal value of inclination angle parameter $K_{\alpha}=K_{\alpha}$ opt is the value of $K_{\alpha}$ at which a particle moves to a specified height in the minimum time. It is determined under the condition of the maximum value of the product $V \cdot K_{\alpha}$. The values of optimal inclination angle parameter $K_{\alpha}=K_{\alpha}$ opt with different $n$ were determined in [4], but as the dependence of $w_{i}$ on parameter $K_{\alpha}$ has been not taken into account here, the results for $n \geq 4$ are incorrect and underestimated. Dependencies of the product $V \cdot K_{\alpha}$ on the inclination angle parameter $K_{\alpha}$ for the different number of normal harmonic amplitudes $n$ are shown in Fig. 4 . The values of optimal inclination angle parameter $K_{\alpha}$ opt , values of the product $V \cdot K_{\alpha}$ and velocity $V$ at $K_{\alpha}=K_{\alpha}$ opt are given in Table 2.

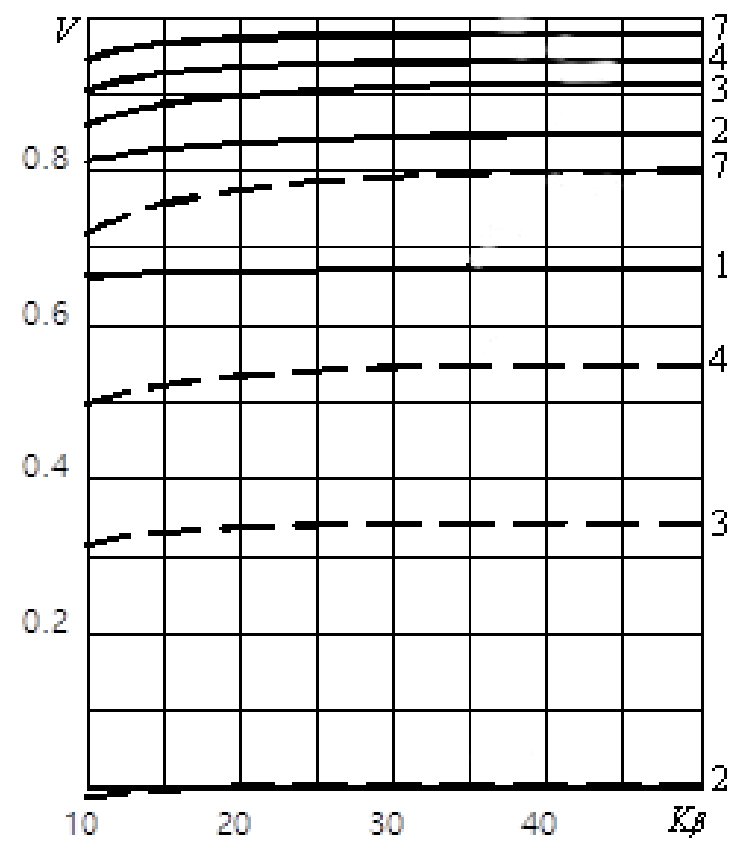

Fig. 3. Dependence of dimensionless velocity $V$ on intensive vibration parameter $K_{\beta}$ with values of parameters $G=1$ at $K_{\alpha}=0$ (continuous lines) and at $K_{\alpha}=0.9$ (dashed lines)

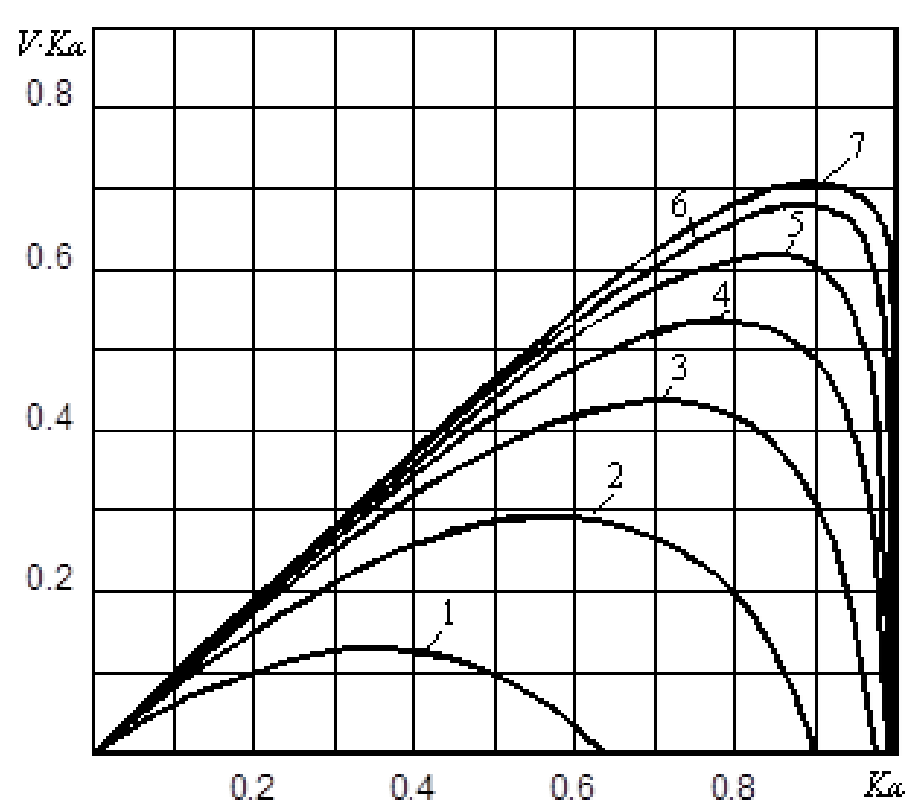

Fig. 4. Dependence of the product $V \cdot K_{\alpha}$ on inclination angle parameter $K_{\alpha}$ with different number of harmonics $n$

\section{Conclusions}

The dimensionless velocity $V$ dependence on dimensionless parameters (the inclination angle parameter and the intensive vibration parameter) for vibratory conveying with harmonic longitudinal and normal polyharmonic vibrations of track for non-hopping modes of parts moving (when parts sliding 
without detachment from the conveying track) is investigated. The limiting and optimal values of the inclination angle parameter have been determined, which allows, knowing the frictional coefficient of moving particles, to determine the maximal and optimal values of the inclination angle of conveying track. The latter is the angle, at which a particle moves to a specified height in the minimum time. The limiting and optimal values of the inclination angle increase with an increase of the number of harmonics.

Velocity $V$ increases with an increase of the number of harmonics $n$, but with each subsequent harmonic this increase decreases. A significant increase of $V$ with an increase of number of higher harmonics is observed only at inclination angles close to the limiting ones. Velocity decreases with the increase of the inclination angle parameter $K_{\alpha}$ (increasing of inclination angle when moving upward). Velocity increases with the increase of intensive vibration parameter $K_{\beta}$ (increasing of longitudinal vibration amplitude), but practically does not change, starting at $K_{\beta}>50 \ldots 80$. The maximum value of velocity is achieved at certain, optimal values of amplitudes of normal vibration harmonics. These values depend on the inclination angle parameter $K_{\alpha}$, with the increasing of parameter $K_{\alpha}$ the optimal values of the lower harmonics $(i \leq n / 2)$ amplitudes increase but values of the higher harmonics $(i>n / 2)$ amplitudes decrease.

For further research, it seems important to create a method for calculating the parameters of polyharmonic normal vibration for practical use in industrial conveyor devices with an electromagnetic drive.

\section{References}

[1] G. Boothroyd, Assembly automation and product design. London, UK: Taylor and Francis Ltd, 2005.

[2] Vibratsiyi v tekhnike: Spravochnik [Vibrations in technics: Reference book], V. N. Chelomey et al., Eds., Vol. 4: Vibratsionnye protsesy i mashyny [Vibration processes and machines], E. E. Lavendel, Ed. Moscow, Russia: Mashinostroyeniye Publ., 1981. [in Russian].

[3] I. Y. Vrublevskyi, "Optymizatsiya parametriv polyharmonichnykh normalnykh kolyvan pid chas bezvidryvnoho vibrotransportuvannia" ["Optimization of parameters of polyharmonic normal vibration in nonhopping vibratory conveying"], Visnyk Natsionalnoho universytety "Lvivska polytekhnika". Optymizatsiya vyrobnychikh protsesiv i tekhnichnyi control u mashynobuduvanni i pryladobuduvanni [Bulletin of Lviv Polytechnic National University. Optimization of Industrial Processes and Technical Control in Mechanical Engineering and Instrument Making], vol. 613, pp. 89-92, 2008. [in Ukrainian].

[4] I. Y. Vrublevskyi, "Optymalni kuty pidyomu shtuchnykh vantazhiv pry bezvidryvnomu vibrotransportuvanni z poliharmonichnymy normalnymy kolyvanniamy" ["Optimal angles of cargo's lifting with non-jumping vibratory conveying by polyharmonic normal oscillations"], Avtomatyzatsiya vyrobnychikh protsesiv u mashynobuduvanni ta pryladobuduvanni [Automation of industrial processes in mechanical engineering and instrumentation], vol. 51, pp. 23-26, 2017. [in Ukrainian].

[5] S. Okabe, Y. Yokoyama, J. Jimbo, "Vibratory conveying by elliptical vibration", Journal of the Japan Society of Precision Engineering, vol. 40, no. 10, pp. 840-845, 1974.

[6] W. A. Morcos, "On the design of oscillating conveyers - case of simultaneous normal and longitudinal oscillations", ASME Journal of Engineering for Industry, vol. 92, no.1, pp. 53-61, 1970.

[7] N. Dallinger, T. Risch, K. Nendel, "Simulation von Förderprozessen bei Vibrationsförderanlagen" ["Simulation of conveying processes in vibratory conveyors"], Logistics Journal: Proceedings, vol. 2012, pp. 1-5, 2012. [Online]. Available: https://www.logistics-journal.de/proceedings/2012/3426/04-dallinger-wgtl2012.pdf. Accessed on: September 30, 2020. [in German].

[8] A. Ishizaka, M. Kimura., T. Kotaki, "The vibratory conveyor applied the vibration involving higher harmonics", Journal of the Japan Society of Precision Engineering, vol. 39, no. 1, pp. 93-98, 1973.

[9] A. V. Dunayevetsky, "Sintez nezavisimykh biharmonicheskikh kolebaniy dlia bezotryvnoho vibrotransportirovaniya" ["Synthesis of independent biharmonic vibrations for non-hopping vibratory conveying"], Avtomatyzatsiya vyrobnychikh protsesiv u mashynobuduvanni ta pryladobuduvanni [Automation of industrial processes in mechanical engineering and instrumentation], vol. 12, pp. 96-99, 1973. [in Russian].

[10] V. G. Yefimov, "Opredeleniye optimalnykh parametrov poliharmonicheskoho poperechnoho zakona dvizheniya lotka" ["Determination of optimal parameters of polyharmonic transverse law of a track's moving"], in Voprosy dinamiki i prochnosti [Problems of dynamics and strength]. Riga, Latvia: Zinatne Publ., vol. 34, pp. 28-38, 1977. [in Russian]. 


\section{Ihor Vrublevskyi}

[11] E. Lavendel, "Sintez optimalnykh vibromashyn" ["Synthesis of optimal vibratory machines"]. Riga, Latvia: Zinatne Publ., 1970. [in Russian].

[12] I. Y. Vrublevskyi, "The phase difference between components of elliptical oscillations of vibratory conveyor providing maximal conveying velocity", Ukrainian Journal of Mechanical Engineering and Materials Science, vol. 1, no. 1, pp. 47-54, 2015.

[13] I. Y. Vrublevskyi, "Optimalnye parametry poliharmonicheskikh normalnykh kolebaniy pri dvukhkomponentnom vibrotransportirovaniyi" ["Optimal parameters of polyharmonic normal oscillations with twocomponent vibratory conveying"], Izvestiya vuzov. Mashinostroyeniye [Proceedings of universities. Mechanical engineering], no. 5, pp. 157-160, 1986. [in Russian]. 